Original Article

\title{
Useful ultrasonographic evaluation of the medial meniscus as a feature predicting the onset of radiographic knee osteoarthritis
}

\author{
Taiki Murakami*, Makoto Enokida, Kei Kawaguchi, Ryoji Otsuki, Hideki Nagashima \\ Department of Orthopedic Surgery, Faculty of Medicine, Tottori University, Yonago, 36-1 Nishimachi, Yonago, Tottori, 683-8504 Japan
}

\section{A R T I C L E I N F O}

\section{Article history:}

Received 16 August 2016

Received in revised form

14 November 2016

Accepted 2 December 2016

Available online 27 December 2016

\begin{abstract}
A B S T R A C T
Objective: Medial radial displacement (MRD) of the medial meniscus is a feature proving a dysfunction in the medial meniscus in osteoarthritis (OA) of the knee. MRD was measured in radiographic pre-OA knee and early osteoarthritis of the knee (early-OA) longitudinally using ultrasound (US) to investigate the characteristics involved in the onset and progression of OA.

Methods: Fifty-five patients with pain on the medial side of the knee participated in the present study. It was possible to follow-up 46 patients for 5 years, and, thus, they were divided into 32 pre-OA patients (female: 59\%, mean age: 69.0 years) and 14 early-OA patients (female: $78 \%$, mean age: 74.4 years) based on radiography at the baseline time-point. MRD was measured in standing and supine positions at baseline and after 1 and 5 years using US. MRD corrected with the skeletal size, i.e., the medial displacement index (MDI), was analyzed. The pre- and early-OA groups were divided into subgroups at 5 years: stable and OA progression groups, following the Kellgren/Lawrence classification, and $\triangle \mathrm{MDI}$ (gap of the MDI between the standing and supine positions) were retrospectively compared between the subgroups at baseline, 1 and 5 years.

Results: In the overall pre-OA group, MDI increased by $7 \%$ and $10 \%$ at 5 years in the supine and standing position, showing a significant increase $(\mathrm{P}=0.044,0.0147) . \triangle \mathrm{MDI}$ was significantly greater in the subgroup with $\mathrm{OA}$ progression in the pre- and early-OA groups $(\mathrm{P}=0.02$ and 0.03 , respectively), and was continuously $6-7 \%$ in the pre-OA progression group, showing that the displacement rate was 2 -fold or higher than in the stable group.

Conclusion: An increase in $\triangle \mathrm{MDI}$ on US may be an important risk factor for the disease stage progression of $\mathrm{OA}$ and useful as a feature predicting the onset of radiographic knee OA.
\end{abstract}

(C) 2016 The Japanese Orthopaedic Association. Published by Elsevier B.V. All rights reserved.

\section{Introduction}

The retention of meniscal function in the knee is closely associated with the onset of knee osteoarthritis (OA) and its aggravation to a more severe state. Its involvement in secondary knee OA with a known cause is clear in many cases: cartilage defects were found to be produced approximately 2 years earlier in patients treated with partial meniscectomy than in age-matched controls, and the risk of knee OA in long-term outcomes was markedly higher in patients treated with meniscectomy than in controls [1,2]. Meniscal degeneration and injury are assumed to be involved in the pathology of primary knee OA, and previous studies reported that the degeneration, tears and positional abnormalities in the meniscus lead to the loss of its main functions, namely, load bearing and

\footnotetext{
* Corresponding author. Fax: +81 859386587.

E-mail address: air-taiki@hotmail.co.jp (T. Murakami).
}

shock absorption, and ultimately to the loss of cartilage [3,4]. Medial radial displacement (MRD) of the medial meniscus is often observed in knee OA [5] and is considered to indicate meniscal dysfunction. The incidence of meniscal malposition in the general population was recently found to be high [6]. Furthermore, meniscal malposition has been identified as a potent risk factor for knee OA [3,7], and MRD is regarded as a part of the pathology involved in the onset and progression of primary knee OA. MRD is more useful when it is evaluated during weight-bearing or the difference between weight-bearing and non-weight-bearing is assessed. Previous findings have been insufficient to conclude the involvement of MRD in the pathology of knee OA because they were obtained using a non-weight-bearing test in their respective studies with MRI. Naredo et al. initially reported meniscal protrusion using US and concluded that US is a non-invasive diagnostic tool for evaluating periarticular and intraarticular abnormalities involved in knee OA [8]. Kawaguchi et al. demonstrated that US is 
capable of evaluating meniscal function in pre-OA and early-stage knee OA (early-OA), which was difficult in previous examinations, because knee OA may be evaluated with and without weight bearing. They concluded that coverage of the joint cartilage surface with the meniscus is similar to that in the normal knee until Kellgren/Lawrence (K/L) radiographic grading system grade 1 , which is pre-OA [9].

Based on the above findings, US appears to be very useful in imaging examinations for the screening of knee $\mathrm{OA}$ including pre-OA and early-OA because it is capable of simply and noninvasively evaluating meniscal function, $[10,11]$. However, previous studies were cross-sectional or short-term studies, and changes with time from pre-OA to early-OA, i.e., the process or characteristics of changes from a functional to dysfunctional meniscus, were not clarified, and, thus, were insufficient to clarify the usefulness of the evaluation of MRD as an index to identify those at risk of developing knee $\mathrm{OA}$.

In the present study, a prospective approximately 5-year followup study was performed using middle-aged-elderly subjects with radiological pre-OA and early-OA in which MRD was measured using US with and without weight bearing. Furthermore, the characteristics of time-course changes in MRD in the knee subsequently developing knee OA and the usefulness of MRD as a risk factor of knee OA were investigated.

\section{Patients and methods}

\subsection{Study design}

Subjects were comprised patients with pre-OA and early-OA. Pre-OA was defined as $\mathrm{K} / \mathrm{L}$ grade 1 or milder $\mathrm{OA}$, and early-OA as $\mathrm{K} / \mathrm{L}$ grade $2 \mathrm{OA}$. In all knees, the $\mathrm{K} / \mathrm{L}$ grade was assessed in a radiographic examination in the standing position and MRD of the medial meniscus was measured in a US examination at the baseline time-point. Regarding patients who discontinued attending the hospital, we contacted them by telephone and set an appointment for re-evaluations one and 5 years after the first investigation. The $\mathrm{K} / \mathrm{L}$ grade was assessed by radiography in the standing position and MRD of the medial meniscus was measured on US in all knees after one and 5 years. Based on the results of the radiographic examination in the standing position after 5 years, subjects were divided into those with and without the progression of $\mathrm{OA}$, namely the progression and stable groups, respectively. The pre- and early-OA groups were retrospectively divided into subgroups: progression and stable groups, and the relationship between MRD and OA progression were investigated. The specific features evaluated are described below.

The Ethics Committee of Tottori University Faculty of Medicine approved the present study. Informed consent was obtained from all participating subjects.

\subsection{Patient selection}

Between February 2007 and March 2012, 720 patients aged 40 years or older visited the outpatient clinic of Tottori University Hospital Department of Orthopedics with knee pain and pain around the knee. Exclusion criteria were knee OA (K/L grade $\geq 3$ ), previous medical histories of fracture around the knee and avascular necrosis, ligament injury of the knee, rheumatoid or other inflammatory arthritis, periarticular fracture, Paget's disease, villonodular synovitis, joint infection, neuropathic arthropathy, acromegaly, hemochromatosis, gout, and pseudo gout, and 55 patients without a previous history of knee surgery who gave consent to the study participated. Forty-six patients for whom it was possible to follow-up for 5 years (mean: 62 months) were selected as subjects. Nine patients were not followed-up because of high tibial osteotomy in 4, partial meniscectomy in 2 and a decline in cooperation with the study in 3. Thirty-four (73.9\%) out of 46 patients were female. On radiography in the standing position at the baseline time-point, pre-OA $(\mathrm{K} / \mathrm{L} \leq 1)$ and early-OA (K/L grade 2$)$ were noted in 32 and 14 knees, respectively, and the latter was medial OA in all cases. Twenty-nine patients had no previous medical history of trauma, and the absence of cartilage defects and apparent meniscal injury (locking and transverse tears in the posterior horn of the medical meniscus) were confirmed on MRI at the baseline time-point. In 17 patients, the absence of meniscal signs and injuries or previous medical histories of cartilage and ligament injuries was confirmed by a physical examination. The absence cartilage loss and meniscal tear were confirmed on MRI within this study period. Among patients with knee pain in or around the unilateral knee, the affected knee was selected, while among those with pain in or around the bilateral knees, the knee with milder symptoms was selected (the right knee: 14 , left knee: 22 ). In the pre-OA group, females accounted for 59\%; the mean age was 69.0 years old and the mean duration of the course observation was 51.3 months. In the early-OA group, females accounted for $78 \%$; the mean age was 74.4 years old and the mean duration of the course observation was 62.0 months.

\subsection{US evaluation}

All patients were subjected to US by 2 examiners (TK and KK) employing the standardized scanning method [12,13].

At the time of the evaluation, the examiners were unaware of whether the subject was a pre-OA or OA subject. US evaluations were performed with both knees in complete extension in both the standing and supine positions. In the standing position, the inclination of the body trunk and pelvis was used as an index to confirm that weight was well balanced on both lower limbs. In the standing position, the feet were placed in a mid-position between the internal and external rotation of the calf. In the supine position, the mid-position was retained by placing retainers on the lateral sides of the feet.

MRD of the medial meniscus was measured in longitudinal US images obtained with a 5-MHz linear transducer (SSD-2000; Hitachi Aloka Medical, Hitachi HI VISION Avius) at a site at which the medial collateral ligament was the most clearly visualized. The medial collateral ligament was visualized as a 3-layer structure on US. The hyperechoic band closest to the superficial layer was the superficial ligament, and the deep ligament was visualized as a hyperechoic band in a deeper layer below hypoechoic bands composed of loose connective tissue. The medial meniscus is typically visualized as hyperechoic tissue located in the center of the femorotibial joint. The margin of the medial meniscus is continuous with the deep medial collateral ligament, and no clear boundary is observed at its attachment site. The femoral condyle, tibia plateau, medial meniscus, and medial collateral ligament may be readily visualized and identified. MRD was measured as the distance from the outermost edge of the medial meniscus to a line connecting the femoral and tibial cortices (Fig. 1).

When marginal osteophytes were present in the femur and tibia, MRD was measured as the clearly visualized normal cortical bone continuous with the femoral and tibial marginal osteophytes. The measurement was performed 3 times in each patient, and the mean was used as the measured MRD value. A magnified image (to an accuracy of $0.1 \mathrm{~mm}$ ) was used to measure MRD on the US monitor.

US measurements of MRD of the medial meniscus were performed at baseline and at the follow-up. The intraobserver intraclass correlation coefficient was $0.959(\mathrm{P}<0.001)$ for the distance 


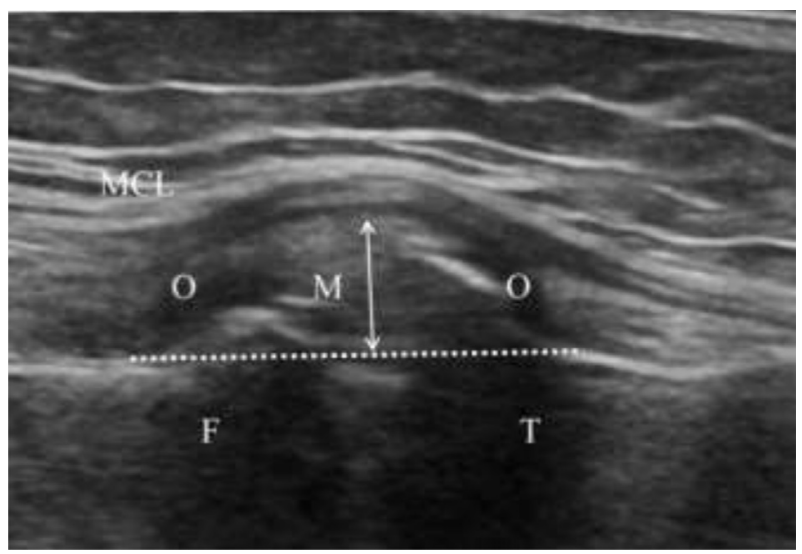

Fig. 1. Longitudinal sonographic image of the right knee of a 66-year-old woman at the level of the medial collateral ligament. Parallel marks running horizontally near the center of the image connect the femoral cortical bone and tibial cortical bone. $\mathrm{MCL}=$ medial collateral ligament; $\mathrm{O}=$ osteophyte; $\uparrow=$ medial radial displacement; $\mathrm{M}=$ medial meniscus; $\mathrm{F}=$ femoral condyle; $\mathrm{T}=$ tibial plateau.

measurement of mMRD. In order to calculate he intraclass correlation coefficient, a single observer examined 10 OA patients, and measurements were performed 3 times at 1 -week intervals. The interobserver intraclass correlation coefficient was 0.987 $(\mathrm{P}<0.001)$ for the distance measurement of mMRD. In order to calculate the intraclass correlation coefficient, three separate observers with different levels of experience in musculoskeletal US examined 10 OA patients.

In addition to measured MRD, the medial displacement index (MDI) was calculated and used for evaluations. The width of the meniscus was corrected for physiques using the following equation reported by McDermott et al. [14]: medial meniscus body width (in $\mathrm{mm})=0.07 \times$ total bony plateau width $+3.70 \mathrm{~mm}$. This method is used to measure meniscal graft sizes, and is simpler to calculate using only radiographs than other reported methods [14-16]. The MRD value was divided by the calculated meniscal width and then multiplied by 100 to yield MDI. The gap of MDI between the standing and spine positions was designated as $\Delta \mathrm{MDI}$.

\subsection{Radiographic evaluation}

All patients were examined by radiography in a double-leg stance at the baseline and at 1 and 5 years (55-63 months). Anteroposterior radiographs of the knee were acquired with both knees fully extended and the subject in a standing position. The $\mathrm{K} / \mathrm{L}$ radiographic grading system was used to measure the global radiographic severity of $\mathrm{OA}$ changes. According to this system, $0=$ normal (no osteophyte), $1=$ possible osteophytes, $2=$ definite osteophytes and possible joint space narrowing, $3=$ moderate/ multiple osteophytes, definite joint space narrowing, some sclerosis, and possible attrition, and $4=$ large osteophytes, marked joint space narrowing, severe sclerosis, and definite attrition [17].

\subsection{Longitudinal evaluation of each group and subgroup}

The OA grade at the baseline was $\mathrm{K} / \mathrm{L}$ grade 2 or milder in patients. Subjects who maintained the $\mathrm{K} / \mathrm{L}$ grade at 5 years were included in the stable group, and those in whom OA progressed by one or more grades were included in the OA progression group. Subjects were subdivided based on the presence or absence of the progression of the OA grade at 5 years: Pre-OA patients were subdivided into those in whom the $\mathrm{K} / \mathrm{L}$ grade was maintained (ppOA group) and progressed to early-OA (>K/L grade 2 ) (peOA group), and early-OA (=K/L grade 2 ) patients were subdivided into those in whom the grade did not progress (eeOA group) and early-OA deteriorated and progressed to K/L grade 2 or more (edOA group). Time-course changes in MRD in the supine and standing positions were investigated in pre-OA knees and early-stage OA knees. Similarly, time-course changes in MDI in each group were evaluated. Regarding $\Delta \mathrm{MDI}$, changes with time were investigated in each subgroup and compared between the groups.

\subsection{Statistical analysis}

A one-way analysis of variance (ANOVA) was used to assess differences in age, body mass index (BMI), and MRD in the supine and standing positions between the groups. In $\triangle \mathrm{MDI}$, a repeated measures ANOVA was used to assess differences between subgroups in each group. When a significant difference was detected by ANOVA, a between (sub) group comparison was performed using Scheffe's test. Additionally, a receiver operating characteristic (ROC) curves was used to determine the cut off $\Delta$ MDI values that were predictors of radiographic OA progression. In all statistical tests, $P$ values less than 0.05 were considered to be significant.

\section{Results}

The baseline characteristics of pre-OA knees and early-OA are presented in Table 1. No significant differences were observed in age, sex, or BMI between the groups.

MRD measured on US was surveyed over time at the baseline, one year, and 5 years (final follow-up). Comparisons of MRD between the supine and standing positions at each time-point in the pre-OA and early-OA groups revealed that it was significantly larger in the standing than in the supine position (Table 2). Regarding changes in MRD with time in the overall pre-OA group, the values obtained were 3.6 and $4.3 \mathrm{~mm}$ in the supine position at

Table 1

Baseline characteristics of OA patients and controls.

\begin{tabular}{lllll}
\hline Group & $\begin{array}{l}\text { No. of } \\
\text { subjects }\end{array}$ & $\begin{array}{l}\text { No. (\%) of } \\
\text { females }\end{array}$ & $\begin{array}{l}\text { Age, } \\
\text { mean } \pm \text { SD } \\
\text { years }\end{array}$ & $\begin{array}{l}\text { BMI, } \\
\text { mean } \pm \text { SD } \\
\mathrm{kg} / \mathrm{m}^{2}\end{array}$ \\
\hline pre-OA & 32 & $19(59)$ & $69.0 \pm 1.0$ & $23.5 \pm 2.9$ \\
early-OA & 14 & $11(78)$ & $74.4 \pm 6.8$ & $25.6 \pm 2.9$ \\
\hline
\end{tabular}

a Differences between osteoarthritis (OA) patients and control subjects were not significant. $\mathrm{BMI}=$ body mass index; $\mathrm{K} / \mathrm{L}=$ Kellgren/Lawrence.

\section{Table 2}

Changes in medial radial displacement of the medial meniscus from baseline to the final follow-up in supine and standing positions. ${ }^{\text {a }}$

\begin{tabular}{llll}
\hline & $\begin{array}{l}\text { MRD in the } \\
\text { supine position } \\
(\mathrm{mm})\end{array}$ & $\begin{array}{l}\text { MRD in the } \\
\text { standing position } \\
(\mathrm{mm})\end{array}$ & $\mathrm{P}$ \\
\hline pre OA(0) & $3.6 \pm 1.0$ & $4.0 \pm 1.1$ & $<0.0001$ \\
pre OA(1) & $4.2 \pm 0.8$ & $4.7 \pm 0.9$ & 0.0022 \\
pre OA(5) & $4.3 \pm 1.2$ & $4.9 \pm 1.3$ & $<0.0001$ \\
e OA(0) & $5.6 \pm 1.0$ & $6.2 \pm 1.1$ & 0.0019 \\
e OA(1) & $6.2 \pm 1.1$ & $6.5 \pm 1.2$ & 0.0022 \\
e OA(5) & $6.2 \pm 1.0$ & $6.6 \pm 1.2$ & 0.0015 \\
\hline
\end{tabular}

${ }^{\mathrm{a}}$ Changes in medial radial displacement (MRD) of the medial meniscus from baseline to the final follow-up in 32 pre-osteoarthritis knees and 14 early-stage osteoarthritis knees, as measured by ultrasound with patients in the supine position as well as in the standing position. Abbreviations: preOA(0), pre-osteoarthritis knees at baseline; preOA(1), pre-osteoarthritis knees at the 1-year follow-up; $\operatorname{preOA}(5)$, pre-osteoarthritis knees at the 5-year follow-up; eOA(0), early-stage osteoarthritis knees at the baseline; eOA(1), early-stage osteoarthritis knees at the 1-year follow-up; eOA(5), early-stage osteoarthritis knees at the 5-year follow-up. Values are the mean $\pm \mathrm{SD}$. 
the baseline and 5 years, respectively, showing a significant increase $(P<0.0001)$. The values obtained in the standing position were 4.0 and $4.9 \mathrm{~mm}$, respectively, also showing a significant increase $(P<0.0001)$. Similarly, changes in MRD in the overall earlyOA group were 5.6 and $6.2 \mathrm{~mm}$ in the supine position at the baseline and 5 years, respectively, showing a significant increase $(\mathrm{P}=0.0012)$, and 6.2 and $6.6 \mathrm{~mm}$ in the standing position, respectively, showing a significant increase $(\mathrm{P}=0.0354)$.

MDI calculated by correcting MRD with the skeletal size was also compared between the standing and supine positions at each time-point in each group (Fig. 2). In the overall pre-OA group, MDI was significantly higher in the standing than in the supine position at all time-points $(\mathrm{P}<0.0001,0.0096,<0.0001)$. Regarding changes with time, MDI increased by $7 \%$ at 5 years in the supine position and $10 \%$ in the standing position, showing a significant increase in both positions ( $\mathrm{P}=0.044,0.0147$ ). In the overall early-stage OA group, MDI was also significantly higher in the standing than in the supine position at all time-points ( $\mathrm{P}=0.0019,0.0022,0.0015)$, whereas no significant increase over that period was noted in either the supine or standing position.

The pre-OA and early-OA groups were divided into subgroups based on the radiographic $\mathrm{K} / \mathrm{L}$ grade in the standing position at 5 years. Pre-OA did not progress in 25/32 (78\%) knees (ppOA), but progressed to early-OA (K/L grade 2 ) in $7 / 32$ (22\%) knees (peOA). Early-OA did not progress in 6/14 (43\%) knees (eeOA), but progressed to OA in $8 / 14$ (57\%) knees (edOA). Changes with time in $\triangle$ MDI representing the gap of MDI between the supine and standing positions were evaluated in each subgroup. The results obtained are shown in Fig. 3. Comparisons between the subgroups revealed that $\triangle \mathrm{MDI}$ was small $(2-3 \%)$ in the ppOA and eeOA groups in which OA did not progress, but was large (6-7\%) in the peOA and edOA groups in which OA progressed, and a significant difference was noted at the baseline between the ppOA and peOA groups and between the eeOA and edOA groups $(\mathrm{P}=0.0289,0.0317) . \Delta \mathrm{MDI}$ significantly increased at 5 years from that at the baseline in the ppOA group ( $P=0.0297$ ); however, no significant change was noted in any other group. ROC curves demonstrated that pre-OA and early-OA $\triangle \mathrm{MDI}$ values were predictors of radiographic OA progression with areas under the curve of 0.783 (95\% confidence interval, 0.572-0.994) in pre-OA group, 0.771 (95\% confidence interval $0.502-1.000$ ) in early-OA group (Fig. 4). In pre-OA, an $\Delta$ MDI greater than $4.83 \%$ had sensitivity of $71.4 \%$ and specificity of $80.0 \%$ for predicting radiographic OA onset. Also, an $\triangle \mathrm{MDI}$ greater than $3.38 \%$ had sensitivity of $50.0 \%$ and specificity of $100 \%$ for predicting radiographic OA progression in early-OA.

\section{Discussion}

US requires no special facility, and is a very useful imaging diagnostic tool for simple and non-invasive imaging examinations of the joints of the four limbs (extremity) [8]. In the knee joint, US is capable of visualizing not only ligament and cartilage injuries, but also meniscal damage, and the joint may be evaluated in real time with and without weight bearing, which makes it the most appropriate imaging diagnostic device for the screening of knee OA [11]. MRD presented in this study is an abnormality of the meniscus detectable by US, and has recently been attracting attention for its involvement in the onset and progression of knee OA [5,12,18]. We initially clarified the characteristic MRD in the knee in which pre$\mathrm{OA}$ progresses to early-OA, and demonstrated that $\triangle \mathrm{MDI}$ may serve as useful features for predicting the onset of knee OA by presenting specific values.

The mechanism of MRD is collapse of meniscal hoop tension caused by the degeneration and tears in the meniscus (tears in the anterior or posterior horn, i.e., radial tears in the posterior segment of the meniscus, which is the anchoring point) [19]. However, no

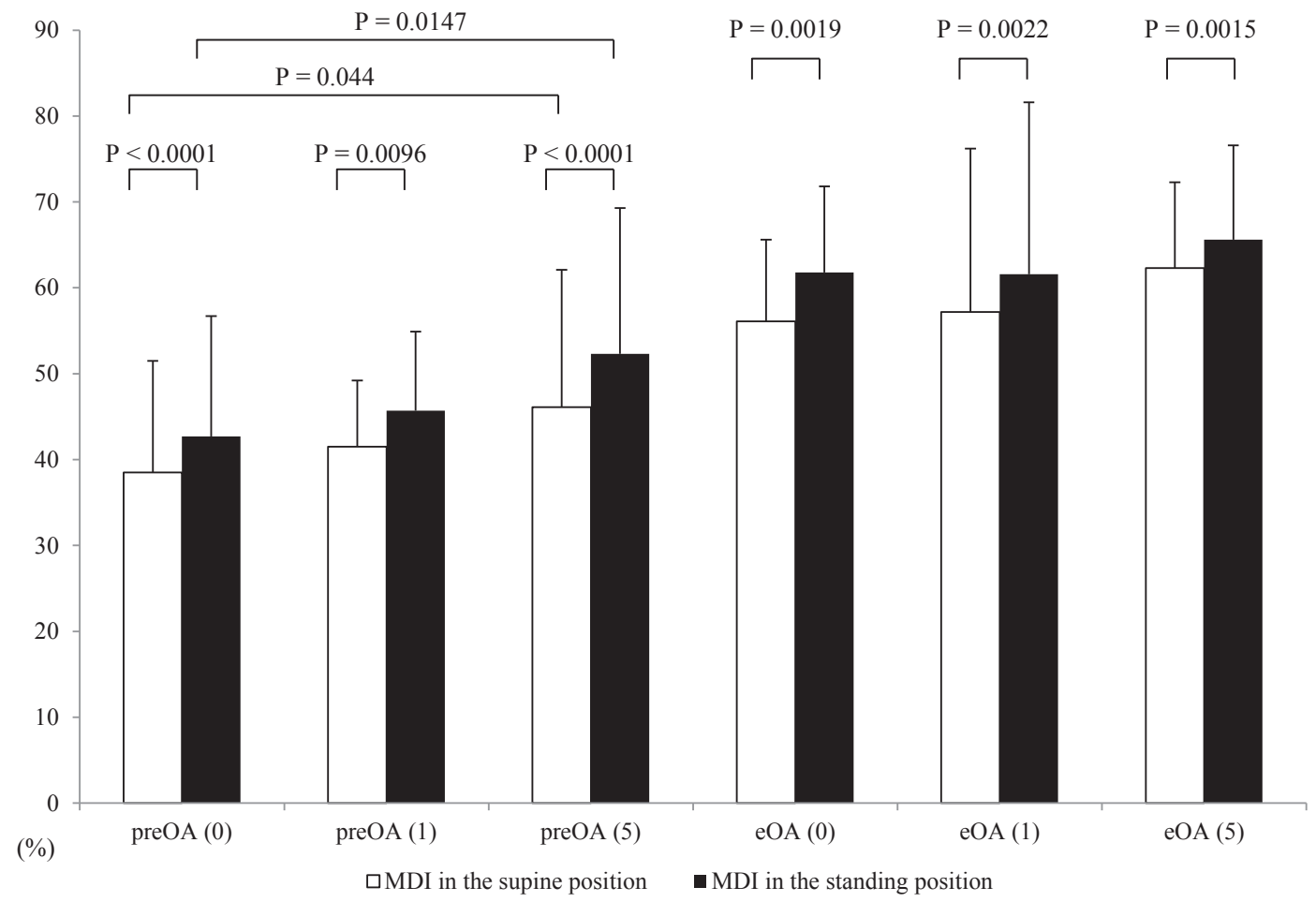

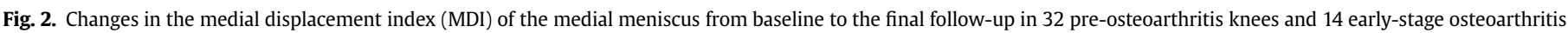

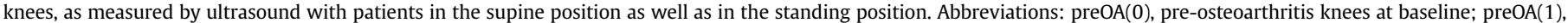

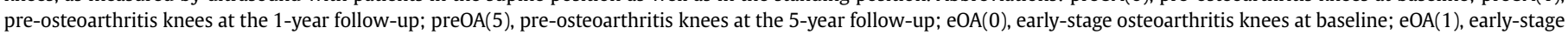
osteoarthritis knees at the 1-year follow-up; eOA(5), early-stage osteoarthritis knees at the 5-year follow-up. Values are the mean \pm SD. 


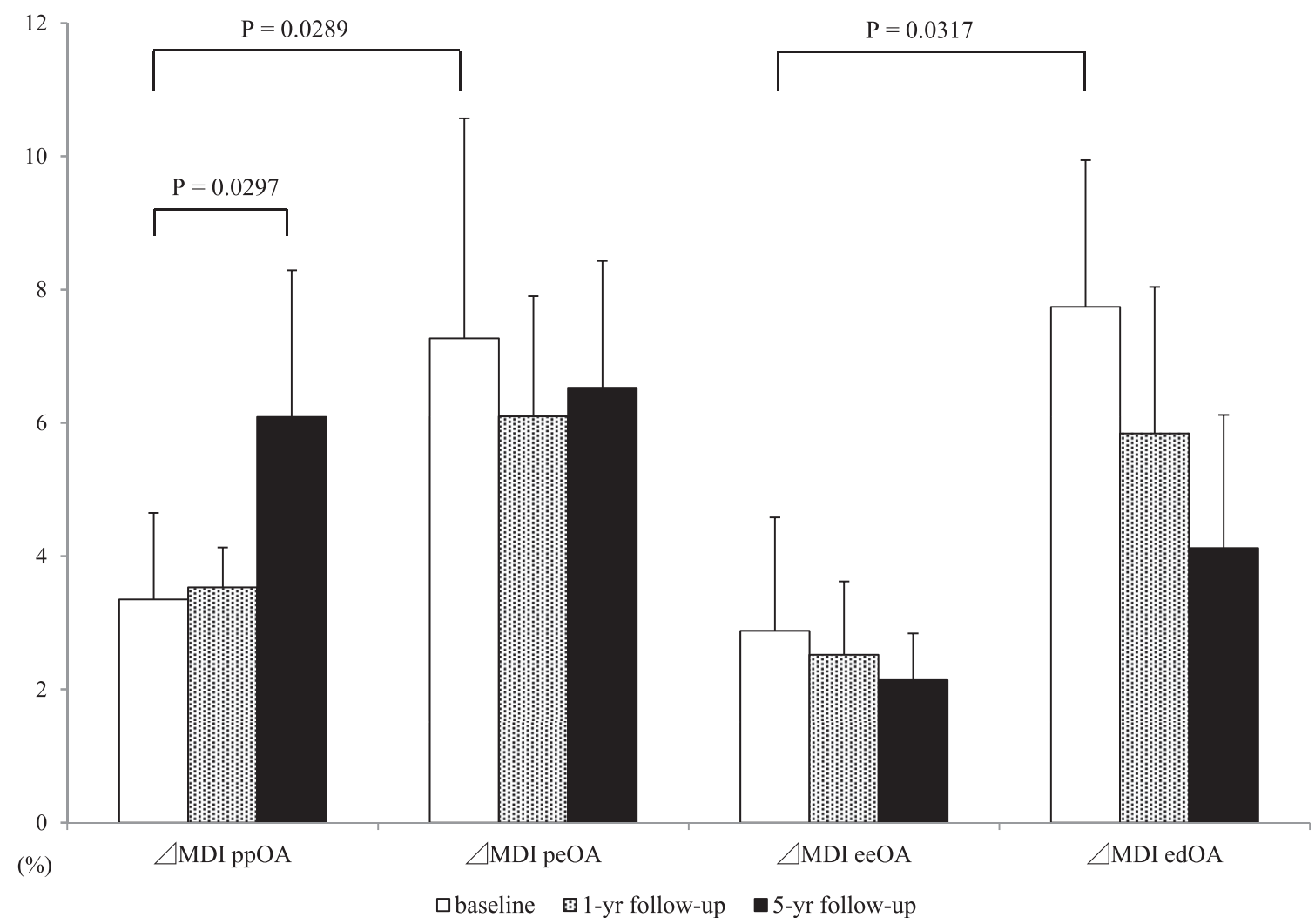

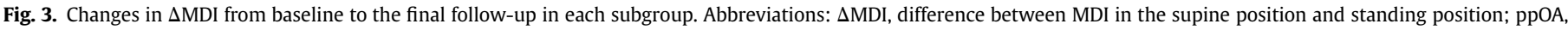

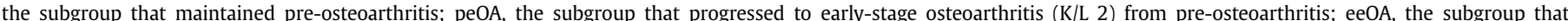

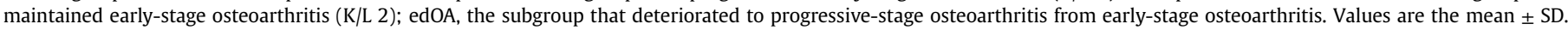

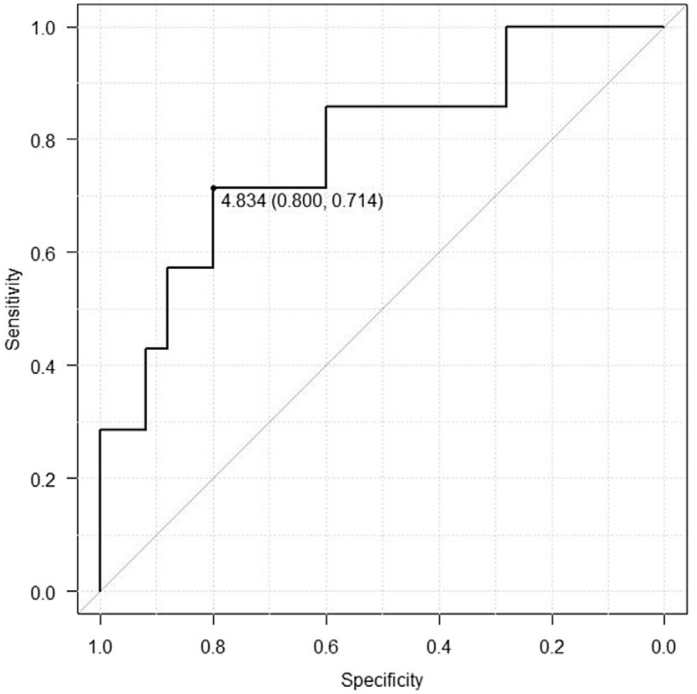

a) pre-osteoarthritis

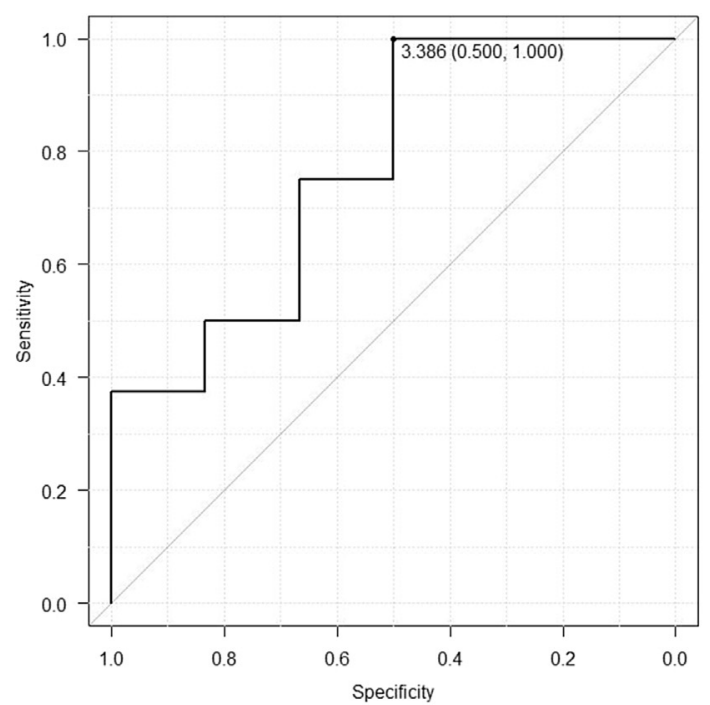

b) early-osteoarthritis

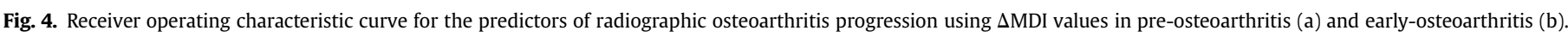

clear meniscal tear is present in MRD in many cases of primary knee OA. Crema et al. reported that meniscal tears were not the only cause of MRD, and independent factors, such as joint cartilage injury and knee varus deformity, play roles [20]. Sugita et al. showed that radial displacement of the medial meniscus preceded narrowing of the medial joint space during the progression of varus osteoarthritis, such that the displaced meniscus is saved from severe degeneration or attrition [12]. Wenger et al. also reported that the medial meniscus extruding to the joint margin showed a triangular to convex shape and was present in a state that escaped tears [21]. Therefore, the increase in MRD observed in primary early-stage knee OA may involve the meniscus morphology and weakened anchoring. In any case, it is valid to consider an increase in MRD as a risk factor for joint destruction because it reduces 
coverage of the joint cartilage surface with the meniscus, thereby increasing peak contact stress loaded on the joint surface, which is followed by the thinning of joint cartilage. Therefore, its involvement in the pathology of knee OA is indisputable.

Follow-up surveys of cartilage defects showed that they may be confirmed even in a 2-year short-term follow-up [22,23]. In a follow-up survey of the $\mathrm{K} / \mathrm{L}$ grade, the $\mathrm{K} / \mathrm{L}$ grade progression rate over approximately 3 years was $22-42 \%$ [24-26]. In the present study, the K/L grade progressed from early-OA (K/L grade 2 ) to 3 in $58 \%$, which is slightly higher than those in preceding studies, and this may have been due to the longer follow-up period, which may have influenced increases in the progression rate. Baseline $\triangle \mathrm{MDI}$ was significantly larger in the edOA group than in the eeOA group, and this may be a risk factor for the progression of OA in advancedstage knee OA. However, osteophyte size has been shown to influence MRD in advanced-stage knee OA, although there are individual variations, and meniscal function is already not normal and is judged to be morphologically abnormal [21]. Thus, in advanced-stage knee OA, it is important that only $\triangle \mathrm{MDI}$ should not be considered to be a primary risk factor for the progression of OA. In other words, an increase of $\triangle M D I$ in advanced-stage knee OA need to be considered as one of the multiple abnormal findings, rather than a predictive factor of the aggravation of $\mathrm{OA}$.

Few studies have been performed on pre-OA pathology from a clinical viewpoint. Primary changes occur in joint cartilage in the onset of knee $\mathrm{OA}$, in which diverse changes occur in the extracellular matrix, such as fibrillation localized in the superficial layer of joint cartilage, a decrease in proteoglycan, cracks in the superficial layer, and the collapse of collagen fiber structures [27]. Therefore, a pathological analysis of the extracellular matrix in the superficial and middle layers of joint cartilage is considered to be more useful than morphological evaluations, such as reductions in joint cartilage thickness and defects, meniscus morphology, and positional abnormalities, for an analysis of the mechanisms underlying the development of early knee OA or an earlier disease stage. However, as described above, studies using US reported that an increase in MRD is related to the severity of OA $[5,7,12,18]$ and that reductions in the weight-sharing function of the extruding meniscus affects subchondral bone [28]. In our 5-year follow-up survey, pre-OA progressed to early-OA (peOA group) in $22 \%$ of subjects, and $\Delta \mathrm{MDI}$ was significantly greater from the beginning of the follow-up in this group than that in the ppOA group; $\triangle \mathrm{MDI}$ was $3.4 \%$ in the ppOA group and $7.2 \%$ in the peOA group, which was significantly greater by more than 2 -fold $(P=0.0289)$. In addition, $\Delta$ MDI $4.83 \%$ was calculated for a cut off values as the risk factor of the radiographic early-OA onset or progression in evaluation by the ROC curve analysis. Consequently, in the radiographic pre-OA knees, $\Delta \mathrm{MDI}>4.83 \%$ increased the risk for onset or progression of early-OA within at least 5 years. We demonstrated the usefulness of US in evaluating knees with and without weight-bearing in real time, and also initially presented abnormal mobility in the meniscus, predicting the progression of pre-OA to early-OA as a specific value. $\Delta$ MDI represents the gap of meniscal extrusion (\%), calculated by dividing MRD by the meniscal width corrected with the skeletal physique, between the standing and supine positions, and is more objective and constant than the value actually measured, $\triangle \mathrm{MRD}$. Since the meniscus morphology is normal in pre-OA unless a meniscal tear is present, there is no major difficulty associated with the correction to MDI. In addition, osteophyte formation is only slight in this disease stage based on the K/L grading system, suggesting that it has a very small influence on the extrusion of the meniscus. We assumed that the increase observed in $\Delta \mathrm{MDI}$ at 5 years in the ppOA group indicated the possibility of progression to early-OA. Based the above results, we herein present $\triangle \mathrm{MDI} 4.83 \%$ as a provisional value of pre-OA likely to progress to $\mathrm{OA}$.
There were several limitations to the present study. The number of subjects was small. This study was performed at a single medical institution (university hospital) in order to avoid variations in the US device and measurement procedure, and subjects were only recruited at the university hospital. Since patients referred for surgery account for most of those at the university hospital, there were fewer patients meeting the criteria of this study during the recruitment period, and some early-OA patients who received conservative treatments at the beginning of the study were subsequently treated with arthroscopic surgery and osteotomy and then dropped out of the study. Furthermore, there were age variations among the subjects. Thus, the age-related influence of the degeneration of knee joint-constituting tissue on MRD was not uniform. However, subjects were pre-OA or early-OA patients, and knees with severe degeneration and injury to the cartilage and meniscus were excluded by MRI examinations in many patients. Therefore, the influence of age may have been small. Another limitation is that the presence or absence of progression to knee OA was not screened by US alone, and plain X-ray images were used to apply corrections with the skeletal physique in order to calculate MDI and $\Delta$ MDI. This may be resolved by verifying the relationship between the tibial joint surface width measured on X-ray and bone widths in many measurable regions by US. Additionally, the middle segment of the medial meniscus was evaluated on US using MCL as an index; however, it was not possible to measure the anterior and posterior segments, and accordingly, we were unable to measure the extrusion of the meniscus in the anteroposterior direction. In many studies using MRI to evaluate MRD, it was mainly examined in the coronal view. There are still no clear evaluation criteria for the meniscus in a sagittal view. Moreover, although US is simple when it is evaluated during weight-bearing, it is difficult to visualize the entire meniscus at the depth reached by US, and an evaluation of meniscus extrusion in the anteroposterior direction is impossible, which is a limitation of the US device.

In conclusion, an approximately 5-year prospective follow-up survey of MRD with and without weight-bearing involving pre$\mathrm{OA}$ and early-OA patients was performed using US. In the knee with radiologically progressing OA, \%MRD difference $(\triangle \mathrm{MDI})$ between the knee with and without weight-bearing significantly increased. In knees evaluated as $\mathrm{K} / \mathrm{L}$ grade 1 or milder showing no abnormal finding on radiography, it is a useful imaging finding that predicts the onset of $\mathrm{OA}$, and the risk factor may be presented as a specific value: $\Delta \mathrm{MDI}>4.83 \%$. Clarification of the relationship between an increase in $\Delta \mathrm{MDI}$ and clinical symptoms in the same period may further verify its pathological significance and lead to preventive treatments for knee OA.

\section{Conflict of interest}

The authors declare that they have no conflict of interest.

\section{Acknowledgments and funding information}

The authors thank Dr. Ryota Teshima for his technical and scientific advice. We also thank Dr. Yasuto Tsukutani and Takahiro Yamashita for their assistance.

\section{References}

[1] Mills PM, Wang Y, Cicuttini FM, Stoffel K, Stachowiak G, Podsiadlo P, Lloyd DG. Tibio-femoral cartilage defect 3-5 years following arthroscopic partial medial meniscectomy. Osteoarthr Cartil 2008 Dec;16(12):1526-31.

[2] Roos H, Lauren M, Adalberth T, Roos EM, Jonsson K, Lohmander LS. Knee osteoarthritis after meniscectomy: prevalence of radiographic changes after twenty-one years, compared with matched controls. Arthritis Rheum 1998 Apr;41(4):687-93. 
[3] Hunter DJ, Zhang YQ, Niu JB, Tu X, Amin S, Clancy M, Guermazi M, Gale D, Felson DT. The association of meniscal pathologic changes with cartilage loss in symptomatic knee osteoarthritis. Arthritis Rheum 2006 Mar;54(3): 795-801.

[4] Fukubayashi T, Kurosawa $H$. The contact area and pressure distribution pattern of the knee: a study of normal and osteoarthrotic knee joints. Acta Orthop Scand 1980 Dec;51(6):871-9.

[5] Kenny C. Radial displacement of the medial meniscus and Fairbank's signs. Clin Orthop Relat Res 1997 Jun;339:163-73.

[6] Englund M, Guermazi A, Gale D, Hunter DJ, Aliabadi P, Clancy M, Felson DT. Incidental meniscal findings on knee MRI in middle-aged and elderly persons. N Engl J Med 2008 Sep 11;359(11):1108-15.

[7] Englund M, Guermazi A, Roemer FW, Aliabadi P, Yang M, Lewis CE, Tomer J Nevitt MC, Sack B, Felson DT. Meniscal tear in knees without surgery and the development of radiographic osteoarthritis among middle-aged and elderly persons: the Multicenter Osteoarthritis Study. Arthritis Rheum 2009 Mar;60(3):831-9.

[8] Naredo E, Cabero F, Palop MJ, Collado P, Cruz A, Crespo M. Ultrasonographic findings in knee osteoarthritis: a comparative study with clinical and radiographic assessment. Osteoarthr Cartil 2005 Jul;13(7):568-74.

[9] Kawaguchi K, Enokida M, Otsuki R, Teshima R. Ultrasonographic evaluation of medial radial displacement of the medial meniscus in knee osteoarthritis. Arthr Rheum 2012 Jan;64(1):173-80.

[10] Riecke BF, Chritensen R, Torp-Pedersen S, Boesen M, Gudberqsen H, liddal H. An ultrasound score for knee osteoarthritis: a cross-sectional validation study. Osteoarthr Cartil 2014 Oct;22(10):1675-91.

[11] Nogueira-Barbosa MH, Greqio-Junior E, Lorenzato MM, Guermazi A, Roemer FW, Chaqas-Neto FA, Crema MD. Ultrasound assessment of medial meniscal extrusion: a validation study using MRI as reference standard. AJR Am J Roentgenol 2015 Mar:204(3):584-8.

[12] Sugita T, Kawamata T, Ohnuma M, Yoshizumi Y, Sato K. Radial displacement of the medial meniscus in varus osteoarthritis of the knee. Clin Orthop Relat Res 2001 Jun;387:171-7.

[13] Freidman L, Finlay K, Jurriaans E. Ultrasound of the knee. Skelet Radiol 2001 Jul;30(7):361-77.

[14] McDermott ID, Sharifi F, Bull AM, Gupte CM, Thomas RW, Amis AA. An anatomical study of meniscal allograft sizing. Knee Surg Sports Traumatol Arthrosc 2004 Mar;12(2):130-5.

[15] Donahue TL, Hull ML, Howell SM. New algorithm for selecting meniscal allografts that best match the size and shape of the damaged meniscus. J Orthop Res 2006 Jul;24(7):1535-43.

[16] Prodromos CC, Joyce BT, Keller BL, Murphy BJ, Shi K. Magnetic resonance imaging measurement of the contralateral normal meniscus is a more accurate method of the determining meniscal allograft size than radiographic measurement of the recipient tibial plateau. Arthroscopy 2007 Nov;23(11): 1174-1179.e1.

[17] Kellgren JH, Lawrence JS. Radiographic assessment osteo-arthrosis. Ann Rheum Dis 1957 Dec;16(4):494-502.

[18] Gale DR, Chaisson CE, Totterman SM, Schwartz RK, Gale ME, Felson D. Meniscal subluxation: association with osteoarthritis and joint space narrowing. Osteoarthr Cartil 1999 Nov;7(6):526-32.

[19] Englund M, Felson DT, Guermazi A, Roemer FW, Wang K, Crema MD, Lynch JA Sharma L, Seqal NA, Lewis CE, Nevitt MC. Risk factor for medial meniscal pathology on knee MRI in older US adults: multicenter prospective cohort study. Ann Rheum Dis 2011 Oct;70(10):1733-9.

[20] Crema MD, Roemer FW, Felson DT, Englund M, Wang K, Jarraya M, Nevitt MC Marra MD, Torner JC, Lewis CE, Guermazi A. Factors associated with meniscal extrusion in knees with or at risk for osteoarthritis: the Multicenter Osteoarthritis study. Radiology 2012 Aug:264(2):494-503.

[21] Wenger A, Wirth W, Hudelmaier M, Noebauer-huhmann I, Trattnig S, Bloecker K, Frobell RB, Kwoh CK, Eckstein F, Enqlund M. Meniscus body position, size, and shape in persons with and persons without radiographic knee osteoarthritis: quantitative analyses of knee magnetic resonance images from osteoarthritis initiative. Arthritis Rheum 2013 Jul;65(7):1804-11.

[22] Wang Y, Ding C, Wluka AE, Davis S, Ebeling PR, Jones G, Cicuttini FM. Factors affecting progression of knee cartilage defects in normal subjects over 2 years. Rheumatology 2006 Jan;45(1):79-84.

[23] Davies-Tuck MLB, Wluka AE, Wang Y, Teichtahl AJ, Jones G, Ding C, Cicuttini FM. The natural history of cartilage defects in people with knee osteoarthritis. Osteoarthr Cartil 2008 Mar;16(3):337-42.

[24] Mazzuca SA, Brandt KD, Katz BP, Lane KA, Buckwalter KA. Comparison of quantitative and semiquantitative indicators of joint space narrowing in subjects with knee osteoarthritis. Ann Rheum 2006 Jan;65(1):64-8.

[25] Lachance L, Sowers MF, Jamadar D, Hochberg M. The natural history of emergent osteoarthritis of the knee in women. Osteoarthr Cartil 2002 Nov;10(11):849-54.

[26] Cooper C, Snow S, McAlindon TE, Kellingray S, Stuart B, Coqqpn D, Dieppe PA Risk factors for the incidence and progression of radiographic knee osteoarthritis. Arthritis Rheum 2000 May;43(5):995-1000.

[27] Pritzker KP, Gay S, Jimenez SA, Osterqaard K, Pelletier JP, Revell PA, van den Berg WB. Osteoarthritis cartilage histopathology; grading and staging. Osteoarthr Cartil 2006 Jan;14(1):13-29.

[28] Wang L, Vieira RL, Rybak LD, Babb JS, Chang G, Krasnokutsky S, Abramson S Reqatte R. Relationship between knee alignment and T1 $\rho$ values of articular cartilage and menisci in patients with knee osteoarthritis. Eur J Radiol 2013 Nov;82(11):1946-52. 